\title{
Alginate Incorporated Multi-Walled Carbon Nanotubes as Dispersive Micro Solid Phase Extraction Sorbent for Selective and Efficient Separation of Acidic Drugs in Water Samples
}

\author{
N. Z. Othman, N. S. M. Hanapi†, W. N. W. Ibrahim and S. H. Saleh \\ Faculty of Applied Sciences, Universiti Teknologi MARA, 40450 Shah Alam, Selangor, Malaysia \\ †Corresponding author: N.S.M. Hanapi; norsuhaila979@uitm.edu.my
}

Nat. Env. \& Poll. Tech.

Website: www.neptjournal.com

Received: 03-09-2019

Revised: $29-10-2019$

Accepted: 07-11-2019

Key Words:

Multi-walled carbon nanotubes Acidic drugs

Micro solid phase extraction Alginate

\begin{abstract}
Innovative development of a simple and rapid dispersive micro solid phase extraction (D- $\mu$-SPE) method combined with high performance liquid chromatography (HPLC) based on alginate incorporated with multi-walled carbon nanotubes (Alg-MWCNT) was developed for the analysis of five selected acidic drugs in the water sample. The effect of dispersive micro solid phase extraction parameters such as the mass of sorbent, sample $\mathrm{pH}$, extraction time and desorption time on the peak area of analytes were optimized. Under the optimum extraction conditions, a linear response was achieved in the concentration range of $1 \mu \mathrm{g} \cdot \mathrm{L}^{-1}$ to $500 \mu \mathrm{g} \cdot \mathrm{L}^{-1}\left(\mathrm{R}^{2} \geq 0.9959\right)$. The limits of detection for the method at a signal to noise ratio of 3 were between $0.03 \mu \mathrm{g} \cdot \mathrm{L}^{-1}$ and $0.08 \mu \mathrm{g} \cdot \mathrm{L}^{-1}$. The proposed method was successfully applied for the determination of four acidic drugs in tap water samples with relative recoveries ranging from 75 $\%$ to $105 \%$. The proposed Alg-MWCNT sorbent showed high potential as an alternative sorbent for dispersive micro solid phase extraction of acidic drugs in aqueous matrices.
\end{abstract}

\section{INTRODUCTION}

In the last few decades, pharmaceuticals have played an increasingly important role in improving the quality of life. Tons of pharmaceutical substances are used in human medicine for diagnosis, treatment or prevention every year (Azzouz \& Ballesteros 2012). The emission of this emerging contaminant as one of the environmental problems may require legislative intervention as they may cause acute effects on flora and fauna even at a low concentration level (Petrovic et al. 2008). The water resource issues in Malaysia have grown in magnitude as the organic contaminant, especially from the disposal of waste, industries and agriculture by-product, may flow and contaminate our water reservoirs (Osman et al. 2012).

Non-steroidal anti-inflammatory drugs (NSAIDs) are among the most frequently prescribed drugs in modern medicine. This group of pharmaceutical drugs provides analgesic (pain-killing) and anti-pyretic (fever-reducing) effect and in higher doses, this drug gives an anti-inflammatory effect (Meek et al. 2010). In this study, a method by using dispersive micro solid phase extraction (D- $\mu$-SPE) for the extraction of these pharmaceutical drugs has been proposed. Model compounds were selected among the pharmaceuticals which are salicylic acid, naproxen, diclofenac, ibuprofen and mefenamic acid.
Pharmaceutical residues are usually present in environmental water samples in trace levels. There are several extraction techniques carried out by the researchers mainly for the isolation and preconcentration of NSAIDs in aqueous matrices such as Solid Phase Extraction (SPE) (Asghari et al. 2016), Solid Phase Microextraction (SPME) (Moeder et al. 2000), Hollow Fiber Liquid-Phase Microextraction (HFLPME) (Sagristà et al. 2010), Stir Bar Sorptive Extraction (SBSE) (Tanwar et al. 2015), Magnetic Solid Phase Extraction (MSPE) (Wang et al. 2017), Dispersive Liquid-Liquid Microextraction (DLLME) (Park \& Myung 2015) and Ultrasound-Assisted Emulsification Microextraction (UAEME) (Lee et al. 2014).

Operating costs, amount of organic solvent used, sample throughput and simplicity of operation frequently becomes the determining factor in choosing the right extraction technique (Saim et al. 1997). However, the most common sample isolation and pre-concentration technique for the extraction of pharmaceuticals in water samples is solid phase extraction (SPE). SPE has been claimed as effective sample preparation for removal of interfering compound and enrichment of analyte (Hennion et al. 1999). Researchers are devoted to developing new sorbents to enhance the extraction process. There were many successful works on the use of different kinds of sorbent materials used in SPE such as molecularly imprinted polymers (MIPs) and carbon nanotubes (CNTs). 
The selection of a suitable SPE extraction sorbent depends on the mechanism of interaction between the sorbent and analyte of interest (Żwir-Ferenc \& Biziuk 2006).

Carbon nanotubes have long been recognized as the stiffest and strongest man-made material (Spitalsky et al. 2010). It possesses advantages in adsorbent-adsorbate interactions but high production cost and non-biodegradable materials (Ren et al. 2011). However, due to its insolubility and creating a homogenous is difficult as it tends to agglomerate caused by strong inter-tube Van der Waals forces (Ibrahim et al. 2015). Besides, because of its nano size, the excellent properties of these structures can only be exploited if they are homogeneously embedded into polymers (Spitalsky et al. 2010). Several types of polymers based on natural products such as cellulose and starch have been proposed in analytical sample preparation due to their biodegradability, good physical and chemical properties and low cost (Hanapi et al. 2017). Many researchers have been working on the use of this sorbent incorporated with biopolymers such as alginate, agarose and chitosan as promising materials to overcome these drawbacks.

Alginate is a biomaterial that has numerous applications in biomedical science and engineering due to its favourable properties, including biocompatibility and ease of gelation (Lee \& Mooney 2012). It offers excellent adsorption due to the presence of hydrophilic and reactive functional groups (Crini 2005). This biopolymer contains a high amount of D-mannuronic acid (M block) and L-guluronic acid (G-block) which can be cross-linked easily by using bivalent ions such as calcium ions to form hydrogels in gelation process (Vijayalakshmi et al. 2016). However, it is easily soluble in aqueous media which limits its application as an adsorbent.

Hence, this research was conducted to investigate the use of MWCNTs incorporated with alginate as a promising material to overcome the disadvantages of these two components with the aid of D- $\mu$-SPE which is believed to be a simple, rapid and efficient method compared to the traditional SPE method.

\section{MATERIALS AND METHODS}

\section{Chemicals and Materials}

Ibuprofen, naproxen, diclofenac, mefenamic acid and salicylic acid were purchased from Sigma-Aldrich (purity assay in range of 98-101\%). Acetonitrile (ACN) and methanol $(\mathrm{MeOH})$ of high performance liquid chromatography (HPLC) grade were purchased from Merck (Darmstadt, Germany). Calcium chloride $\left(\mathrm{CaCl}_{2}\right)$ was obtained from $\mathrm{HmbG}$ Chemicals (Germany). Methane sulfonic acid (MSA) was purchased from Sigma-Aldrich (St. Louis, USA). Ultrapure water was produced from Barnstead Nanopure (Thermo Scientific). Sodium hydroxide and hydrochloric acid were obtained from Merck, Darmstadt, Germany and sodium alginate from Qrec (New Zealand). Multi-walled carbon nanotubes (MWCNTs) with specific surface area $>233 \mathrm{~m}^{2} / \mathrm{g}$, purity $>95 \%, 8-15 \mathrm{~nm}$ outer diameter $\times 50 \mu \mathrm{m}$ in length was purchased from Sun Nanotech (Jiangxi, China).

\section{Preparation of Standard and Sample Solutions}

The individual stock solution of ibuprofen, naproxen, diclofenac, mefenamic acid and salicylic acid were prepared separately in HPLC grade methanol at a final concentration of $1000 \mathrm{mg} . \mathrm{L}^{-1}$. All standard solutions were stored in the amber glass bottle at $4^{\circ} \mathrm{C}$ when not in use. A series of working standard solutions were prepared in methanol by dilution before analysis to prevent from the decomposition of analytes. For calibration standards in the extraction procedure, spiked water samples were prepared by adding $1 \mathrm{~mL}$ of 10 $\mathrm{mg} . \mathrm{L}^{-1}$ standard solutions into $9 \mathrm{~mL}$ of deionized water at a final concentration of $1 \mathrm{mg} \cdot \mathrm{L}^{-1}$.

The river and tap water samples were collected in bottles pre-cleaned with acetone and filtered through a nylon membrane filter to remove colloidal particles and stored in a freezer at $4{ }^{\circ} \mathrm{C}$ until analysis. For accuracy and precision studies, the river and tap water samples $(10 \mathrm{~mL}, \mathrm{pH} 3)$ were spiked with the standard mixture of five pharmaceuticals NSAIDs to give a final concentration of $1 \mathrm{mg} . \mathrm{L}^{-1}$ for each analyte.

\section{Preparation of Alg-MWCNT Composite Beads}

The method used was adapted and modified from literature (Jeon et al. 2010, Sahasathian et al. 2010). The composite beads were prepared by the suspension method. Initially, 3 $\%(\mathrm{w} / \mathrm{v})$ of sodium alginate solution was prepared by stirring it at $60^{\circ} \mathrm{C}$. Meanwhile, $0.3 \mathrm{~g}$ of MWCNT was dispersed in $30 \mathrm{~mL}$ deionized water under sonication for $30 \mathrm{~min}$. Then, the MWCNT solution was added into the sodium alginate solution and sonicated for another hour. The mixture solution was dripped through the injection needle into $1000 \mathrm{~mL}$ of $4 \%$ (w/v) of calcium chloride solution. The Alg-MWCNT beads were formed upon contact with calcium ions. After removal from calcium chloride bath, the beads were rinsed thoroughly with deionized water using an $11 \mu \mathrm{m}$ filter paper and dried in the oven at $50^{\circ} \mathrm{C}$ for 24 hours.

\section{Dispersive Solid Phase Extraction Procedure by Using Alg-MWCNT Sorbent}

About $0.3 \mathrm{~g}$ of Alg-MWCNT powders were dispersed into the aqueous sample $(10 \mathrm{~mL}, \mathrm{pH} 3)$. To trap the analytes, the mixture was vigorously stirred with a magnetic stir bar for 
30 minutes. Subsequently, the Alg-MWCNT powder was isolated from the solution by centrifugation (4000 $\mathrm{r} \mathrm{min}^{-1}$, for $10 \mathrm{~min}$ ). The supernatant was discarded. About $2 \mathrm{~mL}$ of desorption solvent was added, and then the mixture was sonicated for 15 minutes to desorb the analytes. The mixture was then centrifuged at $4000 \mathrm{r} \mathrm{min}^{-1}$, for 5 minutes. The solvent was collected and evaporated to dryness under a soft stream of nitrogen gas. Finally, $10 \mu \mathrm{L}$ of the extract was injected into the HPLC system for analysis. A visual summary of this method is illustrated in Fig. 1. The Alg-MWCNT composite beads could be reused several times after washing with $2 \mathrm{~mL}$ of methanol and water, respectively. Several optimization parameters for D- $\mu$-SPE were optimized by changing one factor at a time.

\section{Chromatographic Conditions}

All analyses were performed using an automated high performance liquid chromatography Dionex Ultimate 3000 (Sunnyvale, CA, USA) system, using an Acclaim Polar Advantage II (5 $\mu \mathrm{m}, 120 \AA, 4.6 \times 150 \mathrm{~mm}$ ) (Dionex USA) as the analytical column. A gradient elution consisting of acetonitrile, 10 $\mathrm{mM}$ methane sulfonic acid (MSA) and ultrapure water was applied. The flow rate was programmed at $1 \mathrm{~mL} \mathrm{~min}^{-1}$. For initialization of the LC analysis, the chromatographic system was cleaned and stabilized for an hour. The quantification of NSAIDs was performed by measuring the peak area of the chromatogram. NSAIDs were detected using a diode array detector at a selected wavelength of $230 \mathrm{~nm}$.

\section{Validation of the Analytical Method}

The extraction method was assessed for linearity $\left(\mathrm{R}^{2}\right)$, limit of detection (LOD), limit of quantification (LOQ), precision (\% RSD) and accuracy (\% relative recovery) before sample analysis. Linearity was measured by plotting the calibration curve of five different concentration levels for the standard mixture of NSAIDs. The same calibration graph was used for the determination of LOD and LOQ. Method precision was measured using three different extractions of calibration standard at the lowest concentration.

\section{RESULTS AND DISCUSSION}

\section{Preparation of Alg-MWCNT Composite Beads}

In the present work, the alginate incorporated multi-walled carbon nanotubes were prepared by the suspension method. Fig. 2 illustrates a mechanism for the formation of alginate incorporated multi-walled carbon nanotubes (Alg-MWCNT). The formations of Alg-MWCNT composite beads start with the carboxylated MWCNTs which were physically combined with sodium alginate which be-came the backbone holding the MWCNTs together. The incorporated Alg-MWCNT structure would form hydrogels immediately after in touch with the calcium chloride solution. This formation occurs due to the active site in a long chain of alginate structure which provides the ability of cross-linked in the presence of divalent ions such as calcium, resulting in the rapid formation
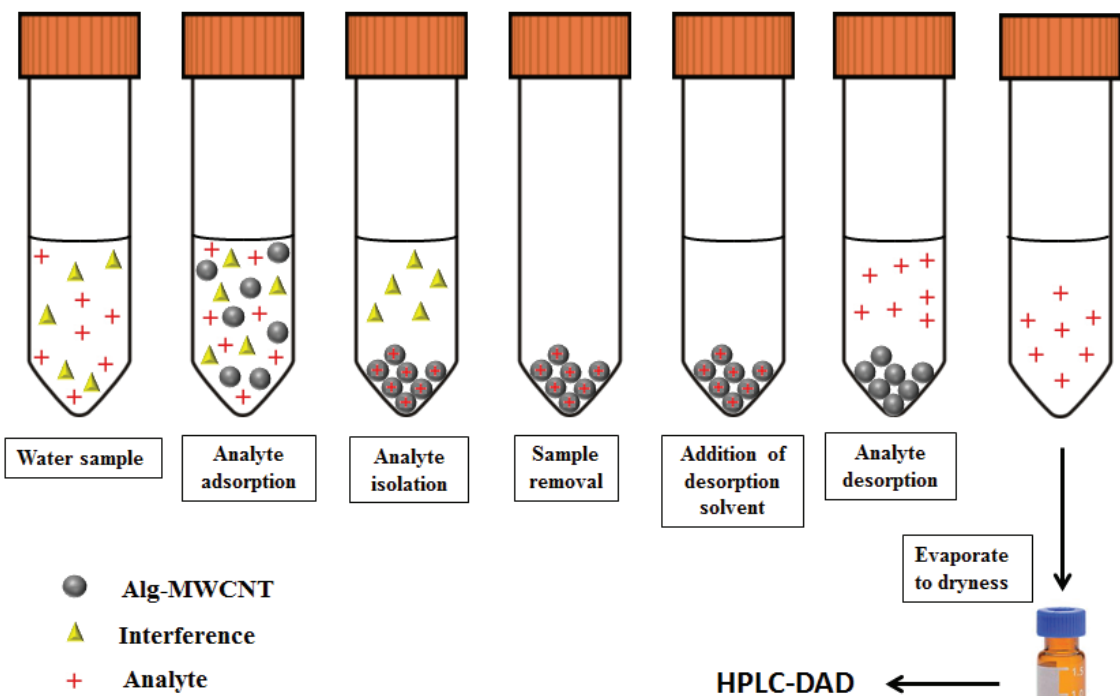

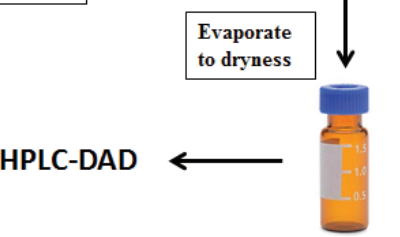

Fig. 1: Dispersive micro solid phase extraction (D- $\mu$-SPE) procedure by using Alg-MWCNT as a sorbent. 


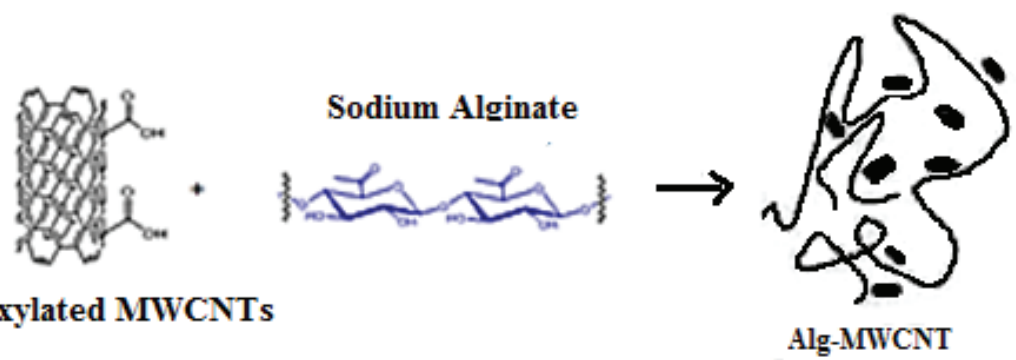

\section{Carboxylated MWCNTs}

Alg-MWCNT

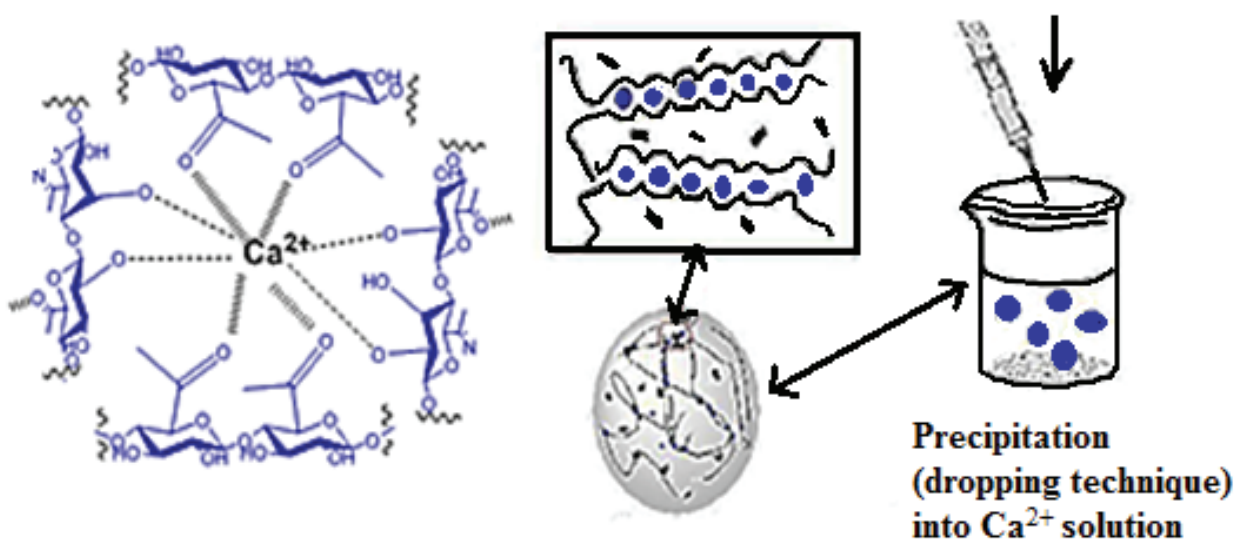

Fig. 2: Mechanism for the formation of Alg-MWCNT.

of hydrogels. The divalent cations preferentially bind toward the G-block rather than M-block (Braccini et al. 1999). The formation of this hydrogels is based on its gel-forming ability through cation binding which was a transition from water soluble sodium alginate into water insoluble calcium alginate (Fertah et al. 2017).

\section{Optimization of Extraction Parameter}

The salicylic acid (SAL), ibuprofen (IBU), naproxen (NAP), diclofenac sodium salt (DIC) and mefenamic acid (MEF) were selected as model analytes in this study as they are the most often used NSAIDs. To determine the optimum conditions for the extraction of NSAIDs from aqueous samples, many parameters that can affect the extraction efficiency were investigated namely extraction time, desorption time, the effect of $\mathrm{pH}$ and mass of sorbent. The optimization was carried out using one variable at a time while other parameters were kept constant. Optimization was carried out using deionized water samples spiked with each NSAID to give a concentration of $1 \mathrm{mg} . \mathrm{L}^{-1}$ in $10 \mathrm{~mL}$ of water samples. About $1 \mathrm{~mL}$ of acetonitrile was used as a desorption solvent. The triplicate extractions were carried out for each parameter optimized in this study.

\section{Effect of Sample pH on Peak Area of Analytes}

The experiment was carried out by varying the $\mathrm{pH}$ value in the range of $\mathrm{pH} 2$ to $\mathrm{pH} 5$ to determine the effect of sample $\mathrm{pH}$ on the extraction of NSAIDs. The sample $\mathrm{pH}$ is expected to significantly influence the efficiency of extraction. According to the $\mathrm{pKa}$ values of salicylic acid, naproxen, diclofenac, ibuprofen and mefenamic acid ( $\mathrm{pKa}=2.98,4.15,4.08,4.91$ and 4.2 respectively), at lower $\mathrm{pH}$ than the $\mathrm{pKa}$ value, these compounds would mostly exist in molecular form. While at higher $\mathrm{pH}$ than the $\mathrm{pKa}$ value, they are in an ionized form.

It was observed that the peak area slightly increased with increasing $\mathrm{pH}$ from $\mathrm{pH} 2$ to $\mathrm{pH} 3$ and highest at $\mathrm{pH} 3$ (Fig. 3a). The peak area starts to decrease after $\mathrm{pH} 3$. As the target compounds were acidic, the $\mathrm{pH}$ of the sample solution was adjusted in the proper acidic range since acidification of an aqueous solution is likely to reduce the dissociation of the weakly acidic analytes which can improve the extraction efficiency (Silva et al. 2008). The extraction was observed to be the best at $\mathrm{pH} 3$, as the sample solution was sufficiently acidic to neutralize the analytes. It has been demonstrated that $\mathrm{pH}$ is a determining condition in the adsorption process of non-steroidal anti-inflammatory drugs onto the sorbent 
and $\mathrm{pH} 3$ is the most suitable condition for the analysis of NSAIDs. Therefore, $\mathrm{pH} 3$ was selected as the best $\mathrm{pH}$ value and used in subsequent experiments.

\section{Effect of Extraction Time on Peak Area of Analytes}

For the optimization of extraction times, in the range of 10 to 50 minutes were employed. The effect of contact time between adsorbent and analytes is shown in Fig. 3b. It was observed that the adsorption capacity of NSAIDs was rapidly increased from 10 to 30 minutes of extraction time. The highest extraction efficiency was obtained at 30 minutes of extraction time. After 30 minutes, the peak area was slowly decreased. This could be due to a long duration of extraction time causes the back extraction of analytes from acceptor into the sample solution (Hernando et al. 2006). Therefore, 30 minutes was selected as the optimal extraction time and used in the subsequent experiments.

\section{Effect of Desorption Time on Peak Area of Analytes}

Ultrasonication method was used for the desorption solvent to desorb NSAIDs from the Alg-MWCNT sorbent as this technique was suitable to be applied for reversible adsorption (Loh et al. 2013). The effect of desorption time in the range of 5-25 minutes of sonication was investigated. It was found that maximum desorption time for the analytes was achieved within 15 minutes of sonication. Beyond 15 minutes of sonication, the peak area of analyte was decreased (Fig. 3c). This is probably due to the heat produced by the sonicator caused the degradation of analyte during the sonication step. Therefore, desorption time of 15 minutes prior to sonication was chosen for subsequent experiments.

\section{Effect of Mass of Sorbent on Peak Area of Analytes}

For the optimization of the mass of sorbent, about $0.1 \mathrm{~g}$ to $0.5 \mathrm{~g}$ of Alg-MWCNT powder was employed. The result showed that the lowest proposed mass of sorbent which is 0.1 g gave the lowest peak area (Fig. 3d). $0.3 \mathrm{~g}$ of sorbent gives the highest peak area of analytes. No significant increase in the peak area was observed with further increase in sorbent mass. The peak areas continue to decrease even with the increase in the mass of sorbent. This might be due to the saturated capacity of MWCNTs as a primary sorption site, thus the excessive amount of sorbent used (more than 0.3 g) resulted in more difficult desorption and required higher volume of desorption solvent (Dahane et al. 2013). Hence, sorbent mass of $0.3 \mathrm{~g}$ was applied for subsequent studies as the peak area for the analytes were at maximum.

\section{Method Validation and Analytical Performance of D- $\mu$-SPE}

The optimization of dispersive solid phase extraction method was then validated for relative recoveries, sample calibration and LOD. Good linearity from the linearity range of 1-500 $\mu \mathrm{g} . \mathrm{L}^{-1}$ was obtained for the four analytes, where the coefficients of determination $\left(\mathrm{R}^{2}\right)$ were in the range of 0.9959 0.9996. The LODs and LOQs were calculated at a signal to noise ratio of 3 and the results were in the range of 0.03-0.08 $\mu \mathrm{g} . \mathrm{L}^{-1}$ for LODs. The precision of the method was measured by the relative standard deviation (RSD) and the results were between 2.6-7.5 for \% RSD. Table 1 shows the validation data for $\mathrm{D}-\mu-\mathrm{SPE}$ of NSAIDs from water samples.

\section{Application of D- $\mu$-SPE on River and Tap Water Samples}

The developed Alg-MWCNT-D- $\mu$-SPE method was successfully applied to river and tap water samples. Relative recovery studies were conducted by spiking the water samples to give a final concentration of $100 \mu \mathrm{g} . \mathrm{L}^{-1}$. The results were tabulated in Table 2. It showed that good relative recoveries were obtained in the range of $75 \%$ to $105 \%$. Thus, dispersive micro

Table 1: Validation data of D- $\mu$-SPE of NSAIDs in water samples.

\begin{tabular}{|llllll|}
\hline Sample & Analytes & Linear range $\left(\mu \mathrm{g} . \mathrm{L}^{-1}\right)$ & Coefficient of determination $\mathrm{R}^{2}$ & $\mathrm{LOD}\left(\mu \mathrm{g} . \mathrm{L}^{-1}\right)$ & Precision $(\mathrm{RSD}, \%)(n=3)$ \\
\hline Tap water & Salicylic Acid & $1-500$ & 0.9989 & 0.054 & 3.2 \\
& Naproxen & $1-500$ & 0.9975 & 0.063 & 5.1 \\
& Diclofenac & $1-500$ & 0.9959 & 0.034 & 3.9 \\
& Ibuprofen & $1-500$ & 0.9996 & 0.080 & 2.7 \\
& Mefenamic Acid & $1-500$ & 0.9994 & 0.078 & 7.5 \\
\multirow{5}{*}{ River water } & Salicylic Acid & $1-500$ & 0.9985 & 0.061 & 4.1 \\
& Naproxen & $1-500$ & 0.9992 & 0.068 & 7.1 \\
& Diclofenac & $1-500$ & 0.9991 & 0.042 & 2.6 \\
& Ibuprofen & $1-500$ & 0.9993 & 0.059 & 5.9 \\
& Mefenamic Acid & $1-500$ & 0.9979 & 0.075 & 5.6
\end{tabular}


Table 2: Relative recoveries (\%) and method precisions (RSD \%, $n=3$ ) of D- $\mu$-SPE in tap water and river water samples.

\begin{tabular}{|c|c|c|c|}
\hline \multirow{3}{*}{ Sample } & \multirow{3}{*}{ Analyte } & \multicolumn{2}{|l|}{ Relative recoveries $(\%)$} \\
\hline & & Spiking level $(n=3)$ & $\operatorname{RSD}(\%)$ \\
\hline & & $100 \mu \mathrm{g} . \mathrm{L}^{-1}$ & \\
\hline \multirow[t]{5}{*}{ Tap water } & Salicylic Acid & 82.7 & 4.2 \\
\hline & Naproxen & 90.1 & 5.9 \\
\hline & Diclofenac & 102.5 & 6.6 \\
\hline & Ibuprofen & 75.3 & 3.8 \\
\hline & Mefenamic Acid & 89.1 & 4.1 \\
\hline \multirow[t]{5}{*}{ River water } & Salicylic Acid & 77.4 & 2.3 \\
\hline & Naproxen & 86.2 & 7.3 \\
\hline & Diclofenac & 105.1 & 6.8 \\
\hline & Ibuprofen & 83.0 & 2.0 \\
\hline & Mefenamic Acid & 97.4 & 3.6 \\
\hline
\end{tabular}

solid phase extraction proved to be an efficient technique for extracting drugs in aqueous matrices. Fig. 4 shows HPLC chromatogram of five NSAIDs which are salicylic acid (SAL), naproxen (NAP), diclofenac (DIC), ibuprofen (IBU) and mefenamic acid (MEF) in river and tap water samples.

\section{CONCLUSION}

In this study, we report the use of alginate incorporated with multi-walled carbon nanotubes for the extraction of acidic drugs using dispersive micro solid phase extraction combined with HPLC-UV. Several parameters were optimized in D- $\mu$-SPE method. The optimum parameters were used in the analysis of real samples. The optimum conditions were

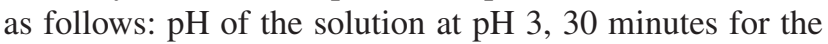
extraction time, 15 minutes for the desorption time and 0.3 $\mathrm{g}$ for the mass of sorbent. All the five analytes which are salicylic acid, naproxen, diclofenac, ibuprofen and mefenamic (a)

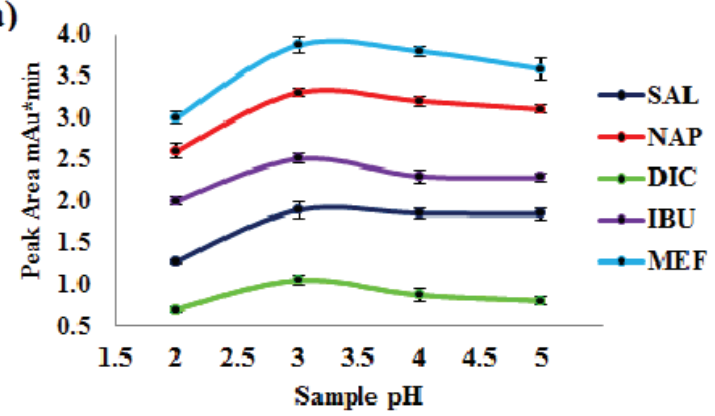

(c)

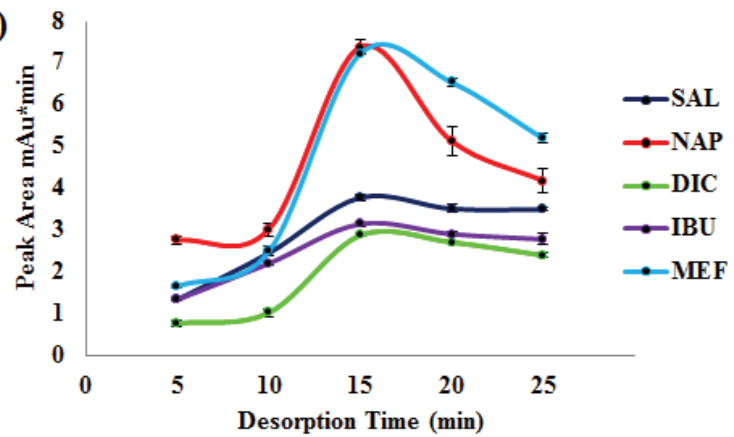

(b)

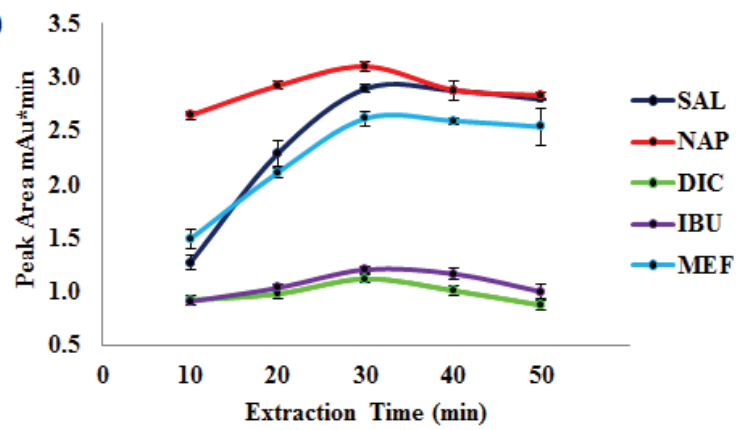

(d)

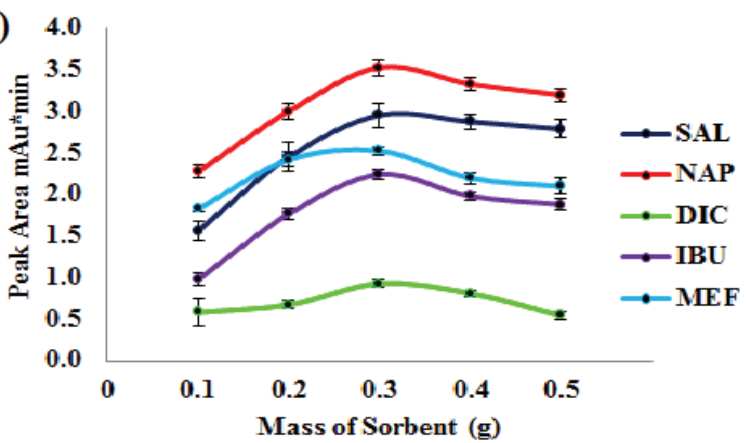

Fig. 3: Optimization for the extraction performance of Alg-MWCNT. (a) Effect of sample pH, (b) extraction time, (c) desorption time and (d) mass of sorbent on Alg-MWCNT-D- $\mu$-SPE of NSAIDs in water samples. (Error bars represent standard deviations of results, $\mathrm{n}=3$ ). 


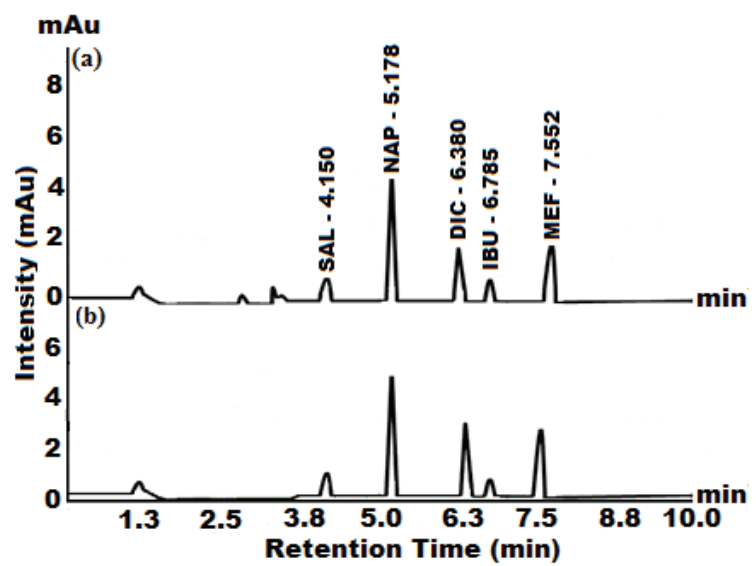

Fig. 4: HPLC chromatogram of five NSAIDs in (a) river water samples and (b) tap water samples.

acid were successfully extracted using the same conditions in D- $\mu$-SPE. Good linearities were achieved for the analytes with coefficients of determination $\mathrm{R}^{2}$, in the range of 0.9959 0.9996. The method was successfully applied to the analysis of river water and tap water samples, with good relative recoveries in the range of $75-105 \%$. Thus, the D- $\mu$-SPE method proved to be a simple, sensitive, selective and green extraction method which could potentially be used in the chemical laboratory for routine analysis of water samples.

\section{ACKNOWLEDGEMENT}

The authors would like to thank Universiti Teknologi MARA for facilitation and the Ministry of Higher Education Malaysia for financial supports through research grant number FRGS 600-IRMI/FRGS 5/3 (010/2019).

\section{REFERENCES}

Asghari, A., Khanalipoor. F., Barfi, B. and Rajabi, M. 2016. Optimized miniaturized air-assisted liquid-liquid microextraction for determination of non-steroidal anti-inflammatory drugs in bio-fluid samples. RSC Adv., 6(111): 109473-109484.

Azzouz, A. and Ballesteros, E. 2012. Combined microwave-assisted extraction and continuous solid-phase extraction prior to gas chromatography-mass spectrometry determination of pharmaceuticals, personal care products and hormones in soils, sediments and sludge. Sci. Total Environ., 419: 208-215.

Braccini, I., Grasso, R.P. and Pérez, S. 1999. Conformational and configurational features of acidic polysaccharides and their interactions with calcium ions: A molecular modeling investigation. Carbohydrate Research, 317(1-4): 119-130.

Crini, G. 2005. Recent developments in polysaccharide-based materials used as adsorbents in wastewater treatment. Progress in Polymer Science, 30(1): 38-70.

Dahane, S., García, M.G., Bueno, M.M., Moreno, A.U., Galera, M.M. and Derdour, A. 2013. Determination of drugs in river and wastewaters using solid-phase extraction by packed multi-walled carbon nanotubes and liquid chromatography-quadrupole-linear ion trap-mass spectrometry. J. Chromatogr. A, 1297: 17-28.
Fertah, M., Belfkira, A., Taourirte, M. and Brouillette, F. 2017. Extraction and characterization of sodium alginate from Moroccan Laminaria digitata brown seaweed. Arabian Journal of Chemistry, 10: S3707-S3714.

Hanapi, N.S.M., Sanagi, M.M., Ismail, A.K., Ibrahim, W.A.W. and Ibrahim, W.N.W. 2017. Ionic liquid-impregnated agarose film two-phase micro-electrodriven membrane extraction (IL-AF- $\mu$-EME) for the analysis of antidepressants in water samples. J. Chromatogr. B, 1046: 73-80.

Hennion, M.C. 1999. Solid-phase extraction: Method development, sorbents, and coupling with liquid chromatography. J. Chromatogr. A, 856(1-2): 3-54.

Hernando, M.D., Mezcua, M., Fernández-Alba, A.R. and Barceló, D. 2006. Environmental risk assessment of pharmaceutical residues in wastewater effluents, surface waters and sediments. Talanta, 69(2): 334-342.

Ibrahim, W.N.W., Sanagi, M.M., Hanapi, N.S.M. and Ibrahim, W.A.W. 2015. Preparation of chemically stable multi-wall carbon nanotubes reinforced blended agarose/chitosan composite film. Der Pharma Chemica, 7(6): 353-356.

Jeon, S., Yun, J., Lee, Y.S. and Kim, H.I. 2010. Removal of $\mathrm{Cu}(\mathrm{II})$ ions by alginate/carbon nanotube/maghemite composite magnetic beads. Carbon Letters, 11(2): 117-121.

Lee, C.H., Shin, Y., Nam, M.W., Jeong, K.M. and Lee, J. 2014. A new analytical method to determine non-steroidal anti-inflammatory drugs in surface water using in situ derivatization combined with ultrasound-assisted emulsification microextraction followed by gas chromatography-mass spectrometry. Talanta, 129: 552-559.

Lee, K.Y. and Mooney, D.J. 2012. Alginate: Properties and biomedical applications. Progress in Polymer Science, 37(1): 106-126.

Loh, S.H., Sanagi, M.M., Ibrahim, W.A.W. and Hasan, M.N. 2013. Multi-walled carbon nanotube-impregnated agarose film microextraction of polycyclic aromatic hydrocarbons in green tea beverage. Talanta, 106: 200-205.

Meek, I.L., Van de Laar, M.A. and Vonkeman, H. 2010. Non-steroidal anti-inflammatory drugs: an overview of cardiovascular risks. Pharmaceuticals, 3(7): 2146-2162.

Moeder, M., Schrader, S., Winkler, M. and Popp, P. 2000. Solid-phase microextraction-gas chromatography-mass spectrometry of biologically active substances in water samples. J. Chromatogr. A. 873(1): 95-106.

Osman, R., Saim, N., Juahir, H. and Abdullah, M.P. 2012. Chemometric application in identifying sources of organic contaminants in Langat river basin. Environ. Monit. Assess., 184(2): 1001-1014.

Park, S.Y. and Myung, S.W. 2015. Simultaneous determination of nonsteroidal anti inflammatory drugs in aqueous samples using dispersive liquid-liquid microextraction and HPLC analysis. Bull. Korean Chem. Soc., 36(12): 2901-2906. 
Petrovic, M., Radjenovic, J., Postigo, C., Kuster, M., Farre, M., De Alda, M.L. and Barceló, D. 2008. Emerging contaminants in wastewaters: Sources and occurrence. In: Emerging Contaminants from Industrial and Municipal Waste, pp. 1-35.

Ren, X., Chen, C., Nagatsu, M. and Wang, X. 2011. Carbon nanotubes as adsorbents in environmental pollution management: a review. Chemical Engineering Journal, 170(2-3): 395-410.

Sagristà, E., Larsson, E., Ezoddin, M., Hidalgo, M., Salvadó, V. and Jönsson, J.Å. 2010. Determination of non-steroidal anti-inflammatory drugs in sewage sludge by direct hollow fiber supported liquid membrane extraction and liquid chromatography-mass spectrometry. J. Chromatogr. A, 1217(40): 6153-6158.

Sahasathian, Teerawat, Praphairaksit, N. and Muangsin, N. 2010. Mucoadhesive and floating chitosan-coated alginate beads for the controlled gastric release of amoxicillin. Archives of Pharmacal Research, 33(6): 889-899.

Saim, N.A., Dean, J.R., Abdullah, M.P. and Zakaria, Z. 1997. Extraction of polycyclic aromatic hydrocarbons from contaminated soil using Soxhlet extraction, pressurised and atmospheric microwave-assisted extraction, supercritical fluid extraction and accelerated solvent extraction. J. Chromatogr. A, 791(1-2): 361-366.

Silva, A.R.M., Portugal, F.C. and Nogueira, J.M.F. 2008. Advances in stir bar sorptive extraction for the determination of acidic pharmaceuticals in environmental water matrices: comparison between polyurethane and polydimethylsiloxane polymeric phases. J. Chromatogr. A, 1209(1-2): $10-16$.

Spitalsky, Z., Tasis, D., Papagelis, K. and Galiotis, C. 2010. Carbon nanotube-polymer composites: Chemistry, processing, mechanical and electrical properties. Progress in Polymer Science, 35(3): 357-401.

Tanwar, S., Di Carro, M. and Magi, E. 2015. Innovative sampling and extraction methods for the determination of nonsteroidal anti-inflammatory drugs in water. J. Pharm. Biomed. Anal., 106: 100-106.

Vijayalakshmi, K., Gomathi, T., Latha, S., Hajeeth, T. and Sudha, P.N. 2016. Removal of copper (II) from aqueous solution using nanochitosan/ sodium alginate/microcrystalline cellulose beads. International Journal of Biological Macromolecules, 82: 440-452.

Wang, T., Liu, S., Gao, G., Zhao, P., Lu, N., Lun, X. and Hou, X. 2017. Magnetic solid phase extraction of non-steroidal anti-inflammatory drugs from water samples using a metal organic framework of type $\mathrm{Fe}_{3} \mathrm{O}_{4} /$ MIL-101 (Cr), and their quantitation by UPLC-MS/MS. Microchim. Act., 184(8): 2981-2990.

Żwir-Ferenc, A. and Biziuk, M. 2006. Solid phase extraction technique-trends, opportunities and applications. Polish Journal of Environmental Studies, 15(5). 